\title{
Numerical simulation of a rectangular clarifier using drift-flux model in OpenFOAM
}

\author{
Muritala Alade Amidu $^{a^{*}}$, Kamal Kayode AbdulRaheem ${ }^{\mathrm{b}}$ \\ ${ }^{a}$ Nuclear Engineering Department, Emirates Nuclear Technology Center, Khalifa University of \\ Science and Technology, Post Office Box 127788, UAE \\ ${ }^{b}$ Automation Department, National Research Nuclear University, Moscow Engineering Physics \\ Institute (MEPHI), Russian Federation \\ a*amidu.alade@ku.ac.ae; ${ }^{\text {k kamalabdulraheem@gmail.com }}$
}

\begin{abstract}
A computation fluid dynamic (CFD) simulation of a rectangular clarifier is performed in this study using a drift-flux model in OpenFOAM CFD code. Using this model with $k-\varepsilon$ turbulence model, the key characteristics (re-circulation and sedimentation) of water-particle mixture flow in a rectangular clarifier are reasonably reproduced. A fairly good agreement is obtained between the simulation results and experimental data of the velocity profiles. Thus, with the demonstrated capability of this CFD model for the prediction of hydrodynamic and sedimentation behavior of water-particle mixture flow, several design issues such as the determination of the best location of baffle in a clarifier can be investigated and addressed. This method can not only provide general conceptual information at the initial design stage but can also be used to perform analysis of different configurations and the effect of changes in operational parameters.
\end{abstract}

Key words: clarifier, drift-flux model, sedimentation, re-circulation, OpenFOAM

\section{Introduction}

Water is one of the essential needs of humans. Water covers a very high percentage of the earth's surface. However, several pollutants contaminate the water, thereby making them impure in their raw form. The pollutants can be solid, liquid, or gaseous substances. Accordingly, several 
techniques have been devised over time to purify water for human needs. The techniques include filtration, distillation, flocculation-coagulation, and sedimentation. The sedimentation technique uses the passive method, gravity, to separate liquid and solid. It is an important technique that is widely applied in chemical, pharmaceuticals, and industrial processes because of its deployment in solid-fluid separation [1][2][3]. Similarly, it has been widely applied for large-scale water treatment for several reasons, including simplicity and lower cost. The technique includes the use of settling tanks that are designed according to rules of overflow rate and detention period based on the assumption of an ideal settling basin [4]. Several other factors such as concentrations or density gradients, wind movement, flow variation, variation of shape of the tank, and in-let and out-let structure are considered to determine the behavior of sediment tanks during operation fully.

Researchers and practitioners have conducted several studies to improve sedimentation techniques. A finite difference method has been used to determine the vertical velocity field and sedimentation distribution of a sedimentation tank [4]. In a similar study, the finite element method is used to establish a relationship between sludge concentration distribution and flow velocity[5]. However, the finite difference method requires very high computation resources, including storage and time. Therefore, a more efficient technique that used a Strip Integral Method (SIM) has been applied to solve the governing differential equations of continuity, momentum, and mass transport[6]. Similarly, the spatial and temporal development of influent particle size distribution toward the out-let of a rectangular sedimentation basin is described with a computer simulation according to a fundamental governing mechanism of particle growth and motion. The general performance of the settling basin is predicted based on the model[7].

A study was conducted on cohesive sediment and the process of settling in a sedimentation tank [8]. In the study, a numerical investigation of three-dimensional settling process using CFD-DEM 
(Computational Fluid Dynamics-Discrete Element Method) considering inter-particle collision force, the van der Waals force, and the fluid-particle interaction forces was carried out to establish the exact influence of the characteristics of sediment particles such as the Bond number and particle size distribution on cohesive features. It was found that the cohesive silt characteristics in the settling process depend on the cohesion among silt particles and the particle polydispersity. Ghawi and Josef carried out a study with the objective to improve the operational performance of large horizontal rectangular sedimentation tanks. They applied a full two-dimensional mass conservative model, which is based on computational fluid dynamics, to realize their objectives by analyzing and modeling the two-phase flow regime found in settling tanks and comparing the model with experimental data [6]. A CFD package FLUENT 6.3.26 was used to develop the novel CFD code technique that is used to analyze sediment transport for multiple particle sizes in sedimentation tanks of potable water.

Recently, Long Fan et al. studied the flow dynamics in a secondary sediment tank. A computational flow dynamics technique was used to establish the velocity profile and the solids concentration distribution in the tank [9]. The study further established the influence of the position of baffle in the secondary sedimentation tank. Similarly, an overflow rate technique was used to remove fine particles from a tertiary sedimentation tank. In the study, an adjustable baffle, which is inclined at different angles, was used to examine particle removal efficiency in the tank [10]. The study revealed that the sedimentation tank with an adjustable baffle at different angles is more effective for particle removal. Another study compared Lamella plate design, often called inclined plates, and a conventional design to examine sedimentation efficiency in a rectangular tank. The result indicated an improved sedimentation efficiency by the Lamella plate design relative to a conventional design [11]. 
Several numerical methods have been previously used to predict the behavior and performance of a settling tank. However, most of these CFD methods involve high computational cost. Thus, a simple drift-flux model which is an alternative mathematical formulation to the described methods in the previous section is used in the present work to capture both the flow and sedimentation characteristics of a rectangular settling tank to demonstrate the accuracy of the new CFD method proposed for the evaluation of the settling tank performance. Consequently, the challenge of high computational resources is solved.

\section{Governing equations of the drift-flux model}

Generally, for the type of multiphase flow problem described in the preceding section where solid particles are dispersed in a liquid. If the liquid is assumed to be continuous while the solid is considered dispersed within the liquid, the dynamics of the system can be comprehensively described by the conservation equations (continuity, momentum, and concentration) for each of the phases (liquid and solid) with appropriate closure relations. To reduce the number of conservation equations to be solved, an alternative mathematical formulation of the problem has been proposed and it is called the mixture model. With this mixture model, only one continuity equation and one momentum equation are written for the mixture of solid and liquid phases, instead of writing separate equations for each phase. The mixture of the two phases is considered as single phase. Thus, the mixture flow is described by the following governing equations of mass, momentum, particle volume fraction, and energy in Eq. (1), Eq. (2), Eq. (3), and Eq. (4), respectively.

$$
\frac{\partial \rho_{m}}{\partial t}+\nabla \cdot\left(\rho_{m} u_{m}\right)=0
$$


$\frac{\partial\left(\rho_{m} u_{m}\right)}{\partial t}+\nabla \cdot\left(\rho_{m} u_{m} u_{m}\right)=-\nabla P+\nabla \cdot\left(\mu_{m} \nabla u_{m}\right)+\nabla \cdot\left(\alpha \rho_{p} u_{p m} u_{p m}+(1-\right.$

$\left.\alpha) \rho_{b f} u_{b f m} u_{b f m}\right)+\rho_{m} g_{k}$

$\frac{\partial\left(\rho_{p} \varphi\right)}{\partial t}+\nabla \cdot\left(\varphi \rho_{p} u_{m}\right)=-\nabla \cdot\left(\varphi \rho_{p} u_{p m}\right)$

where $u_{m}, u_{p m}, u_{b f m}, \rho_{p}, \rho_{b f}$ represent the mixture velocity vector, relative velocity of particles, relative velocity of the base fluid (water), density of particles, the density of the base fluid, and mixture temperature, respectively. The gravity vector is denoted by $g_{k}$. Additionally, the physical properties of the mixture: dynamic viscosity, and density are represented by $\mu_{m}$, and $\rho_{m}$ which are defined as shown below in Eqn. (5) based on the viscosity correction model for generic power-law plastic and Eqn. (6), respectively.

$\mu_{m}=\mu_{b f}+0.00023143 *\left(10^{179.26 \varphi}-1\right)$

$\rho_{m}=(1-\varphi) \rho_{b f}+\varphi \rho_{p}$

The most important phenomenon in settling tanks employed for water treatment is the sedimentation of particles in the water in the tank. As stated previously, the sedimentation or settling of particles causes the slip motion of particles. Therefore, the relative velocity of particles and base fluid resulting from the settling of particles is assumed to follow the Vesilind sedimentation model [12] given by Eq. (6) and Eq. (7).

$$
\begin{aligned}
& u_{p m}=\frac{\rho_{b f}}{\rho_{m}} u_{0} 10^{-A \varphi} \\
& u_{b f m}=\frac{\alpha \rho_{p}}{(1-\alpha) \rho_{f}} u_{p m}
\end{aligned}
$$


where $u_{0}$ and $A$ represents the reference settling velocity vector and settling coefficient which can be determined from the experimental data. In this study, the settling coefficient, $A=285.84$ and $u_{0}=(0,-0.002198 \mathrm{~m} / \mathrm{s}, 0)$ (values recommended by Vesilind).

To capture the physics of particle slip motion resulting from the sedimentation of particles in the base fluid (water in this case), a mixture model implemented in OpenFOAM CFD code version 6 is used in this study. The basic assumptions of this two-phase mixture modeling approach are: the particles and the base fluid are in local thermal equilibrium, the slip motion of particle due to sedimentation is considered and physical properties of the mixture are evaluated based on the local concentration of the particles. As a developmental basis, the drift flux solver (driftFluxFoam) implemented in OpenFOAM 6 is used to capture the mixture modeling concept.

\section{Table 1. Physical properties of water and solid particles}

\begin{tabular}{ccc}
\hline Properties & Water & Solid Particles \\
\hline Density, $\rho, \mathrm{kg} / \mathrm{m}^{3}$ & 998.2 & 3600 \\
Dynamic viscosity, $\mu, \mathrm{kg} / \mathrm{ms}$ & 0.0001003 & - \\
\hline
\end{tabular}

\section{Problem description, boundary conditions and numerical scheme}

Flows in a settling tank is a two-phase turbulent flow which is characterized by the sedimentation of the solid particles in the flow field. Figure. 1(b) shows the geometry of the computation domain used in this study, which is the simplification of the experimental setup of Imam [4]. This geometry is typical of the settling zone and outlet zone of rectangular clarifier as can be seen in Fig. 1(a). Although, flow in settling tank is generally three-dimensional, however, a two-dimensional is 
considered in this study because the inlet and outlet are separated in such a way to uniformly spread the width of the tanks, which makes the three-dimensional effect insignificant. The computation domain (see Fig. 1(b)) is bounded by the following surfaces with defined dimensions: the free surface top wall $\left(D_{1}=73 \mathrm{~cm}\right)$, outlet surface $\left(D_{2}=2 \mathrm{~cm}\right)$, baffle height $\left(D_{3}=7 \mathrm{~cm}\right)$, inlet height $\left(D_{4}=5\right.$ $\mathrm{cm})$ and the bottom wall $\left(\mathrm{D}_{5}=73 \mathrm{~cm}\right)$.

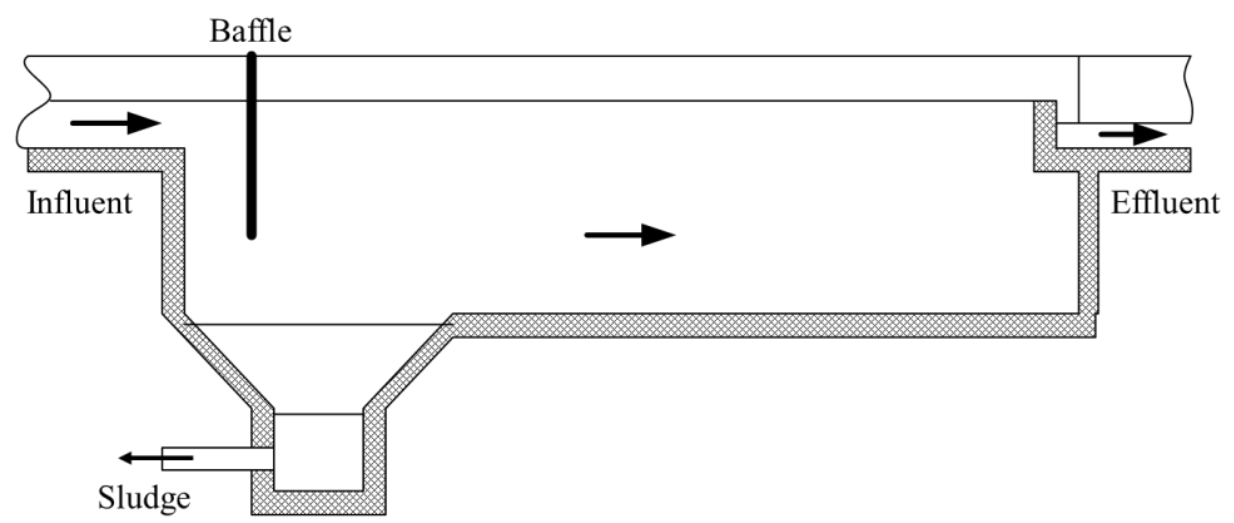

(a)

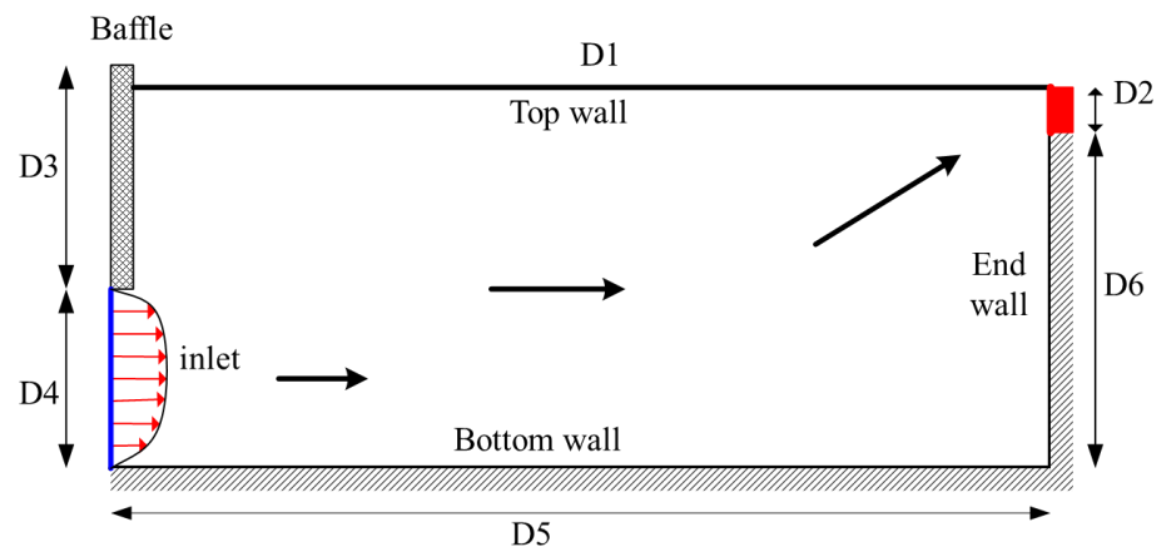

(b)

Figure 1. Two dimensional geometrical configuration of the rectangular sedimentation tank: (a) Horizontal flow rectangular clarifier, (b) Idealized solution domain A slip boundary condition is imposed on the free surface represented by the top wall to account for the effect of winds and small ripples on the flow field. The other surfaces in the geometry are 
considered as non-slip boundaries for the velocity. For calculation involving the volume fraction equation, zero particle flux boundary conditions (Neumann boundary condition) in place of constant value boundary conditions (Dirichlet boundary condition) are imposed on all sides. The standard $\boldsymbol{k}-\boldsymbol{\varepsilon}$ turbulent model is used with the standard wall functions. A uniform hexahedral mesh of 14000 cells (optimized) is used after the performance of grid sensitivity tests. An initial concentration of solid particles is assumed to be $0.1 \%$ while the base fluid is assumed to be water. The physical properties of the water-particle mixture are obtained using the Eqn. (4) and Eqn. (5) for mixture viscosity and density, respectively.

A PIMPLE algorithm of OpenFOAM CFD code is used in the solver to obtain the solution of the governing equations described in Eqn. (1), Eqn. (2), and Eqn. (3). Additionally, Gauss linear,

Gauss linear corrected and Gauss linear spatial numerical schemes are used for the solution of gradients, Laplacian, and divergence in the governing equations. Moreover, an adaptive time step which is based on the maximum Courant number $\left(C_{o}\right)$ in the computational domain is used with the restriction on the Courant number $\left(C_{o} \leq 0.5\right)$. This has not impact in this study since the steadystate calculation is sought. Thus, the absolute residuals of the key variables (velocity, temperature, pressure, and particle concertation) are restricted to the maximum of $10^{-6}$ as the convergence criterion of the iteration process of the PIMPLE loop.

\section{Discussion of simulation results}

To validate the capability of the drift-flux mixture model for the prediction of the hydrodynamic and sedimentation characteristic of a rectangular clarifier, an experimental test case with constant inlet velocity of $0.024 \mathrm{~m} / \mathrm{s}$ and Reynolds number of 10900 is considered in this study. The sedimentation of the suspended solid particles is reasonably captured by the sedimentation sub- 
model of the drift-flux model as shown in Fig. 2(a). With the suspended solid particles injected with the water at the inlet at $0.1 \%$ concentration, the predicted particle distribution shows the gradual settling of the particles at the bottom of the clarifier as the computation progresses with time until a steady-state solution is reached which is shown in Fig. 2(a).

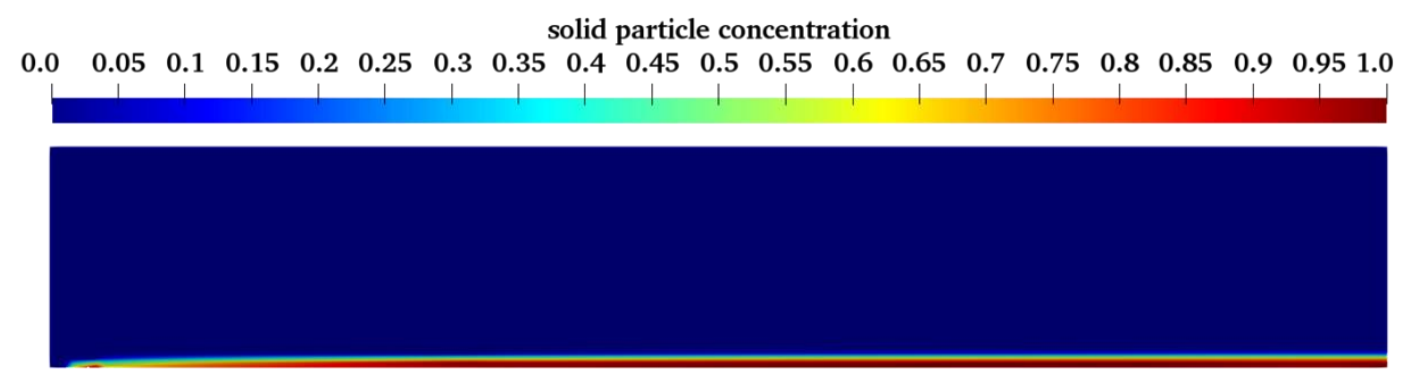

(a)

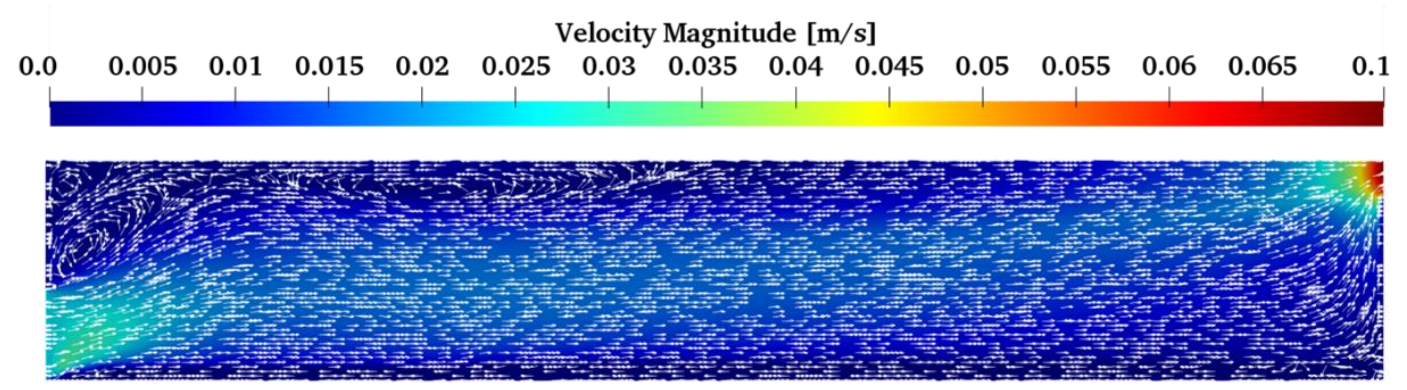

(b)

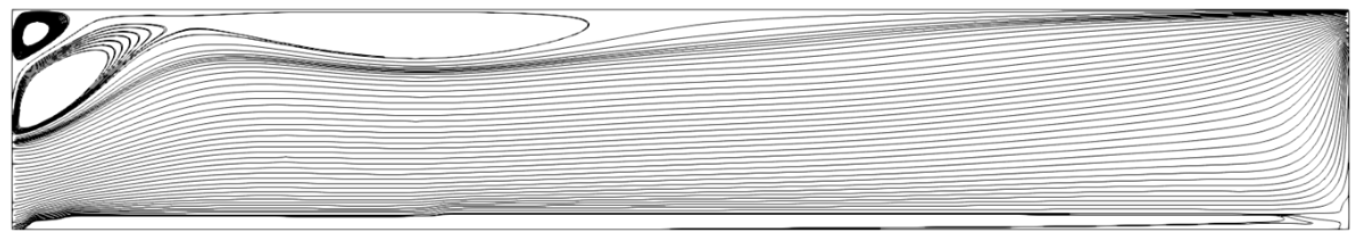

(c)

Figure 2. Qualitative simulation results: (a) Particle concentration distribution; (b) velocity contours; (c) flow streamline shown fluid recirculation

In addition, the hydrodynamic behavior of the water-particle mixture is also reasonably captured by the model. This is indicated by the flow filed shown in Fig. 2(b) where the expected recirculation of flow at the top left corner can be seen in accordance with the experimental 
observation. The recirculation flow is accentuated by velocity streamline shown in Fig. 2(c). In fact, the predicted reattachment length is about $0.4 \mathrm{~m}$ which compares fairly well with the experimental data of $0.38 \mathrm{~m}$. This means that the $k-\varepsilon$ turbulence model used in this study is able to reproduce the turbulence behavior of the suspended particles laden fluid flow. The vortices in the inlet zones trap particles, efficiently forming sludge blankets where they can flocculate further or simply recirculate until they move into a lower velocity region from which they can settle out.

A more quantitative comparison of the simulation results and experimental results is shown in Fig. 3 in which the velocity profiles at different position along the horizontal length of the rectangular clarifier are compared. The positions along the horizontal length considered are: $\mathrm{X}=0084 \mathrm{~m}, 0.252$ $\mathrm{m}, 0.336 \mathrm{~m}$, and $0.50 \mathrm{~m}$. Based on the comparison done in Fig. 3, it can be said that the drift-flux mixture model is able to predict the entire flow field in terms of the velocity profiles reasonably well.

\section{Conclusion}

The computational fluid dynamic model (drift-flux mixture model) described in this study can substantially reproduce both the hydrodynamic and sedimentation behavior of water-water mixture flow in rectangular clarifier. With this model, several design issues such as the determination of the optimal location of baffle in the rectangular clarifier can be investigated and addressed at reasonable computational cost since it provides simplification (through reduction of conservation equations) of the full Eulerian multiphase model without compromising the model for key phenomena in clarifiers. This CFD method can also be used for the optimization of the design to achieve robust and stable operation of the sedimentation tank under divergent conditions given the economics, increasing availability of water and environmental responsibility. 


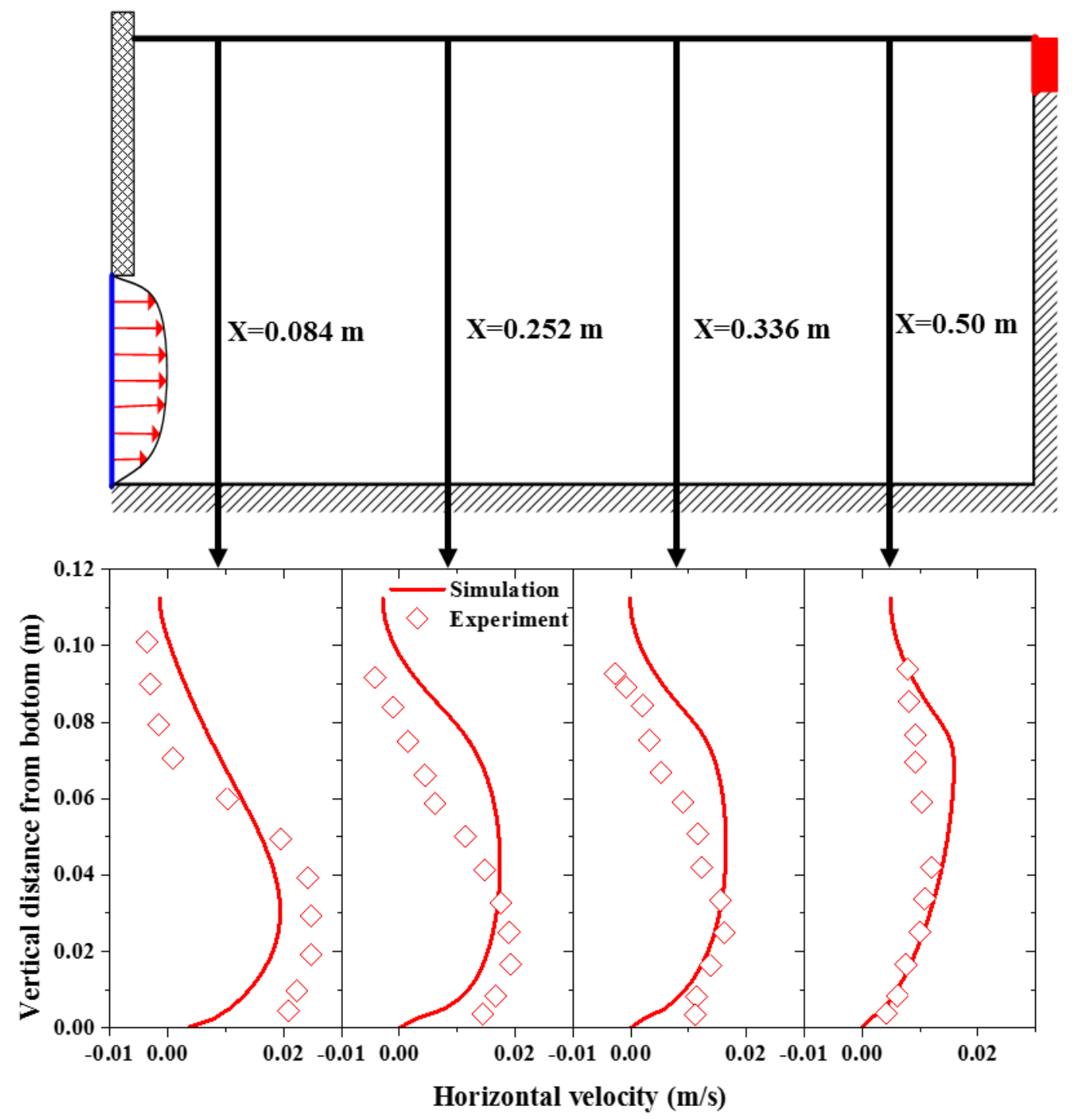

Figure 3. Comparison of longitudinal velocity distribution between the simulation results and experimental data at different horizontal location $(X=0.084 \mathrm{~m}, 0.252 \mathrm{~m}, 0.336 \mathrm{~m}$, and $0.50 \mathrm{~m})$ in the settling tank

\section{References}

[1] Bürger R, Wendland WL. Sedimentation and suspension flows: Historical perspective and some recent developments. J Eng Math 2001;41:101-16.

https://doi.org/10.1023/A:1011934726111. 
[2] Di Felice R. The sedimentation velocity of dilute suspensions of nearly monosized spheres. Int J Multiph Flow 1999;25:559-74. https://doi.org/10.1016/S03019322(98)00084-6.

[3] Komiwes V, Mege P, Meimon Y, Herrmann H. Simulation of granular flow in a fluid applied to sedimentation. Granul Matter 2006;8:41-54. https://doi.org/10.1007/s10035005-0220-3.

[4] Imam E, McCorquodale JA, Bewtra JK. Numerical Modeling of Sedimentation Tanks. J Hydraul Eng 1983;109:1740-54. https://doi.org/10.1061/(asce)07339429(1983)109:12(1740).

[5] Tulus, Suriati, Situmorang M, Zain DM. Computational Analysis of Sedimentation Process in the Water Treatment Plant. J Phys Conf Ser 2017;890:0-5. https://doi.org/10.1088/1742-6596/890/1/012077.

[6] Hadi GA, Kriš J. A CFD methodology for the design of rectangular sedimentation tanks in potable water treatment plants. J Water Supply Res Technol - AQUA 2009;58:212-20. https://doi.org/10.2166/aqua.2009.027.

[7] Valloulis IA, List EJ. Numerical Simulation of a Sedimentation Basin. 1. Model Development. Environ Sci Technol 1984;18:242-7. https://doi.org/10.1021/es00122a006.

[8] Sun R, Xiao H, Sun H. Investigating the settling dynamics of cohesive silt particles with particle-resolving simulations. Adv Water Resour 2018;111:406-22. https://doi.org/10.1016/j.advwatres.2017.11.012.

[9] Fan L, Xu N, Ke X, Shi H. Numerical simulation of secondary sedimentation tank for 
urban wastewater. J Chinese Inst Chem Eng 2007;38:425-33.

https://doi.org/10.1016/j.jcice.2007.06.006.

[10] Guo H, Ki SJ, Oh S, Kim YM, Wang S, Kim JH. Numerical simulation of separation process for enhancing fine particle removal in tertiary sedimentation tank mounting adjustable baffle. Chem Eng Sci 2017;158:21-9.

https://doi.org/10.1016/j.ces.2016.09.022.

[11] Tarpagkou R, Pantokratoras A. The influence of lamellar settler in sedimentation tanks for potable water treatment - A computational fluid dynamic study. Powder Technol 2014;268:139-49. https://doi.org/10.1016/j.powtec.2014.08.030.

[12] Vanderhasselt A, and Vanrolleghem P.A. Estimation of sludge sedimentation parameters from single batch settling curves, Water Research, 34(2000) 395-406, https://doi.org/10.1016/S0043-1354(99)00158-X

\section{Declarations}

\section{Availability of data and materials}

Data and materials can be provided on a reasonable request.

\section{Competing interests}

The authors declare that they have no known competing financial interests or personal relationships that could have appeared to influence the work reported in this paper

\section{Funding}

Not applicable 


\section{Authors' contribution}

Muritala A Amidu: Conceptualization, Methodology, Validation, Writing. Kamal K Abldul

Raheem: Software, Resources, writing - review \& editing

\section{Acknowledgments}

Not applicable

6. Author's information (optional) 